\title{
The Living Atlases community in action: general introduction
}

Marie-Elise Lecoq $q^{\ddagger}$, Anne-Sophie Archambeau ${ }^{\ddagger}$, Fabien Cavière ${ }^{\ddagger}$, David Martin§, Nick dos Remedios§

‡ UMS PatriNat - GBIF France, Paris, France

$\S$ Atlas of Living Australia - CSIRO, Canberra, Australia

Corresponding author: Marie-Elise Lecoq (melecoq@gbif.fr)

Received: 04 Apr 2018 | Published: 21 May 2018

Citation: Lecoq M, Archambeau A, Cavière F, Martin D, dos Remedios N (2018) The Living Atlases community in action: general introduction. Biodiversity Information Science and Standards 2: e25487.

https://doi.org/10.3897/biss.2.25487

\section{Abstract}

Atlas of Living Australia (ALA) (https://www.ala.org.au/) is the Global Biodiversity Information Facility (GBIF) node of Australia. In 2010, they launched an open and free platform for sharing and exploring biodiversity data. Thanks to this new infrastructure, they have been able to drastically increase the number of occurrences published through the GBIF.org . In order to help other GBIF nodes or institutions, they made all of their modules publicly available for reuse and customization through GitHub (https://github.com/ AtlasOfLivingAustralia).

Since 2013, the community created by developers interested by ALA tools, organized, with the help of GBIF, 8 technical workshops around the world. These workshops helped the launch of at least 13 data portals. The last training session, funded through the GBIF Capacity Enhancement Support Programme (https://www.gbif.org/programme/82219/ capacity-enhancement-support-programme), was been attended by 23 participants from 19 countries on 6 continents. Moreover, on the new GBIF website, a section has been dedicated to this programme (https://www.gbif.org/programme/82953/living-atlases), the Living Atlases community official website has been launched in 2017 (https://livingatlases.gbif.org) and the technical documentation has been improved and translated in several languages. All of these achievements would not have been possible without a huge effort from the ALA developer community. 
After a brief introduction of the Living Atlases community, we will present you the work done by ALA to simplify the process of getting a living atlas up and running. We will also show you how ALA developers managed to help the community members to create their own version by performing simple HTML/CSS customizations.

\section{Presenting author}

Marie-Elise Lecoq

Nick dos Remedios 